\title{
Inverse Airfoil Design Utilizing CST Parameterization
}

\author{
Kevin A. Lane* and David D. Marshall ${ }^{\dagger}$ \\ California Polytechnic State University, San Luis Obispo, CA, 93407-0352
}

\begin{abstract}
An inverse airfoil design process is presented that makes use of the CST parameterization method. The CST method is very powerful in that it can easily represent any airfoil shape within the entire design space of smooth airfoils. This makes it an ideal modeling technique for an inverse design process because accurate airfoil geometry treatment is required. The downfall of some inverse design processes is that they do not accurately handle the leading edge region due to large flow gradients and high curvature distributions. One way to account for this is by representing airfoils with smooth analytic functions, such as the CST method. The inverse airfoil design process presented is based on the relation between pressure residuals and the required airfoil shape change. The pressure residuals give the sign of the normal vector with which to modify the airfoil shape. The CST method is then used as the smoothing algorithm. The inverse design method is simple, accurate, and efficient. It is shown to accurately determine the airfoil geometry in both subsonic and transonic flows. Since this method simply examines pressure distributions to modify the airfoil shape, the flow solver can be kept separate from the inverse design process, allowing any fidelity flow solver to be used.
\end{abstract}

\section{Nomenclature}

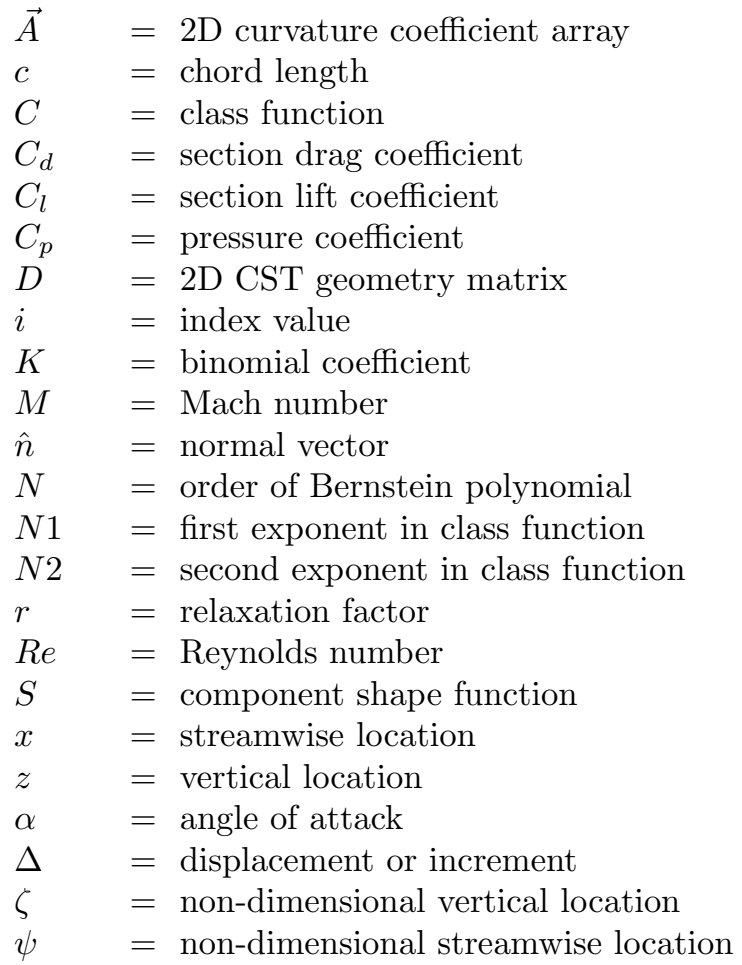

*Graduate Student, Aerospace Engineering Department, Student Member AIAA

${ }^{\dagger}$ Associate Professor, Aerospace Engineering Department, Senior Member AIAA 


\begin{tabular}{|c|c|c|}
\hline \multicolumn{3}{|c|}{ Subscripts } \\
\hline$L$ & & lower surface \\
\hline$o$ & $=$ & objective or target value \\
\hline$U$ & $=$ & upper surface \\
\hline$x$ & & streamwise location \\
\hline$z$ & & vertical location \\
\hline$\infty$ & $=$ & freestream value \\
\hline
\end{tabular}

\section{Introduction}

ERODYNAmic inverse design methods are very powerful in their efficiency. They are much more comAputationally efficiency than a direct optimization approach because the desired performance is already specified by a pressure or velocity distribution. It does not have to be found by a search technique. However, this is also a downside to the method. The designer is left with the task of creating pressure distributions that reflect the design goals. It can be difficult to ensure that the selected pressure distribution has minimal drag for the desired performance. Inverse design methods do not find the optimal performance, they simply get as close to the desired performance as possible. Target pressure optimization methods have been created to help relieve the designer of this task. ${ }^{1-2}$

There are many aerodynamic inverse design methods available for either airfoil or wing design. The residual-correction methods such as Takanashi's ${ }^{3}$ and NASA's streamline curvature method ${ }^{4}$ are popular inverse design methods. Both employ an iterative correction of either pressure or velocity differences along the target and designed airfoil surfaces. More recently, $\mathrm{Yu}^{5}$ presented an inverse design method based on streamline equations that is both efficient and accurate. This new method does not make the assumption of streamline curvature variations normal to the airfoil surface that the streamline curvature method does. It instead contains a small perturbation geometric equation that is deduced from the streamline momentum equations, the continuity equation, as well as the isentropic relations including the geometry similarity assumption of near streamlines to the airfoil surface.

$\mathrm{Yu}$ points out that "aerodynamic characteristics are very sensitive to airfoil leading-edge geometry, and its accurate treatment is a limitation of many existing design methods." The leading-edge geometry can be more accurately handled through the design method or the geometry modeling. Yu has already shown that the inverse design method based on streamline equations can accurately determine the leading-edge shape for both subsonic and transonic cases.

Since airfoil modifications in inverse design methods do not produce smooth airfoils, a smoothing technique is required. A geometric parameterization method that represents airfoils with smooth analytic functions can be utilized to accomplish this. Kulfan's class/shape transformation (CST) ${ }^{6}$ parameterization method defines a basic shape with the class function and modifies it to the desired shape with the shape function. The CST method can efficiently model any airfoil in the entire design space. This capability makes it attractive for aerodynamic design methods and is why CST was selected for airfoil smoothing.

\section{Inverse Airfoil Design}

For inverse airfoil design, a target pressure distribution and an initial airfoil shape are specified. The pressure distribution of the initial airfoil is compared to the target pressures, and modifications are made to the initial airfoil according to the selected inverse design method. These modifications result in an airfoil that has a pressure distribution more closely resembling the target pressure distribution. Inverse design methods are commonly tested by specifying the target pressure distribution as that of a known airfoil. As the pressure distribution converges to the target distribution, the airfoil should also converge to the target airfoil. Figure 1 shows an example of initial and target airfoils. The initial airfoil is a NACA 0012 and the target airfoil is an RAE 2822. To reach the target airfoil, the leading edge and lower aft region of the initial airfoil must be perturbed with a negative outward

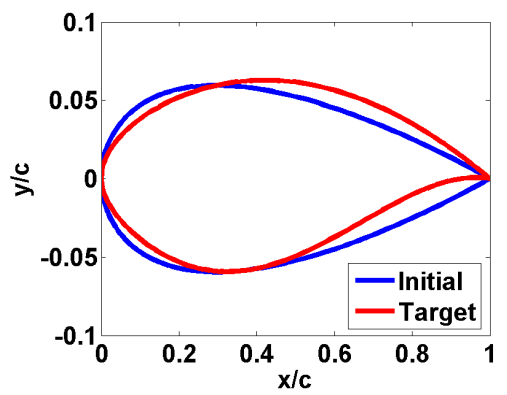

Figure 1. Initial and Target Airfoils facing normal and the upper aft region must be perturbed with a positive outward facing normal. 
Figure 2 shows initial and target pressure distributions for different Mach numbers and angles of attack. The initial and target airfoils are again a NACA 0012 and an RAE 2822 respectively. The pressures at the leading edge and lower aft region of the initial airfoil are all less than those of the target airfoil. Also, the pressures at the upper aft region of the initial airfoil are greater than those of the target airfoil. Therefore, wherever the pressure is greater than the target pressure $\left(C_{p}-C_{p o}>0\right)$, the airfoil surface must be perturbed with a positive outward facing normal vector. Conversely, wherever the pressure is lower than the target pressure $\left(C_{p}-C_{p o}<0\right)$, the airfoil must be perturbed with a negative outward facing normal.

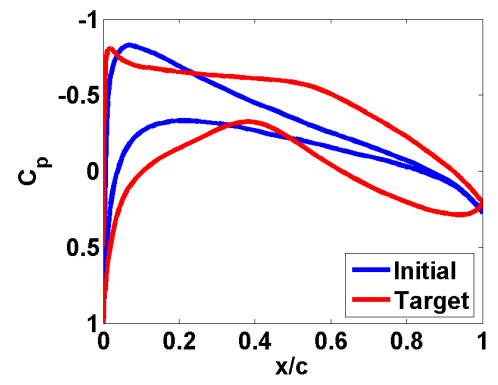

(a) $M_{\infty}=0.6, R e=1.0 \times 10^{7}$, and $\alpha=$ $1.5^{\circ}$

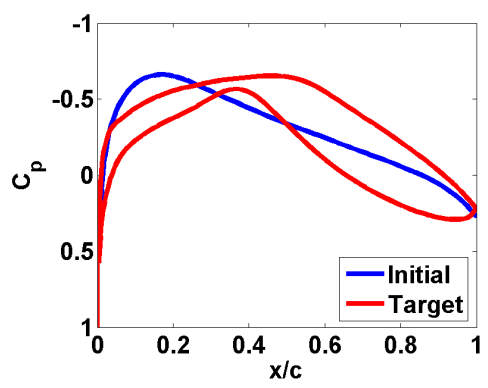

(b) $M_{\infty}=0.725, R e=1.0 \times 10^{7}$, and $\alpha=0.0^{\circ}$

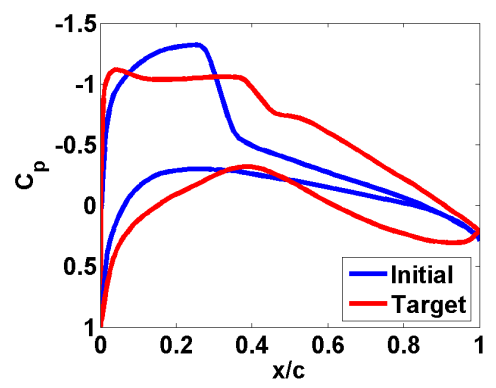

(c) $M_{\infty}=0.715, R e=1.0 \times 10^{7}$, and $\alpha=2.3^{\circ}$

Figure 2. Initial and Target Pressure Distributions

This gives way to a straightforward method to modify the airfoil shape in an inverse design process. At each design iteration, the pressure residuals can be used to control the normal vector along which each point is perturbed by. As previously mentioned, the pressure residuals and the required normal perturbation direction have the same sign. Therefore, the direction is given by the pressure residuals and the magnitude each point is moved along its normal vector simply needs to be scaled appropriately. The perturbations used to modify the airfoil are expressed as:

$$
\Delta \hat{n}(x)=\frac{\left(C_{p}(x)-C_{p o}(x)\right)}{r} \cdot \hat{n}(x)
$$

where $\mathrm{r}$ is a relaxation factor to control convergence and ensure the airfoil is not perturbed enough to yield a non-realistic airfoil. Each airfoil coordinate is then perturbed by that magnitude along its normal vector to yield the updated airfoil:

$$
\begin{aligned}
x_{i+1} & =x_{i}+\Delta \hat{n}_{x} \\
z_{i+1} & =z_{i}+\Delta \hat{n}_{z}
\end{aligned}
$$

Since this will not result in a smooth airfoil, a smoothing process must be implemented. The updated airfoil coordinates are parameterized using the CST algorithm. This produces a smooth airfoil to be used in the next iteration of the inverse design. Since this method simply examines pressure distributions to obtain the updated airfoil, it will work for any fidelity model.

\section{CST Parameterization}

The CST method is a powerful parameterization technique for modeling both two-dimensional and threedimensional shapes. Since CST was used for airfoil smoothing, this paper will limit the discussion to the two-dimensional CST equations. Any smooth airfoil can be represented by the general two-dimensional CST equations. The only things that differentiate one airfoil from another in the CST method are two arrays of coefficients that are built into the defining equations. These coefficients control the curvature of the upper and lower surfaces of the airfoil. This method of parameterization captures the entire design space of smooth airfoils and is therefore useful for any application requiring a smooth airfoil. This work builds on earlier work by Lane and Marshall ${ }^{7}$ where CST was used in a direct airfoil optimization scheme. 


\section{III.A. 2D CST Introduction}

The CST method is based on Bezier curves with an added class function. The upper and lower surface defining equations are as follows:

$$
\begin{aligned}
& \zeta_{U}(\psi)=C_{N 2}^{N 1}(\psi) \cdot S_{U}(\psi)+\psi \cdot \Delta \zeta_{U} \\
& \zeta_{L}(\psi)=C_{N 2}^{N 1}(\psi) \cdot S_{L}(\psi)+\psi \cdot \Delta \zeta_{L}
\end{aligned}
$$

Equation (3) uses the following non-dimensional coordinates:

$$
\begin{aligned}
& \psi=x / c \\
& \zeta=z / c
\end{aligned}
$$

The last terms in Eq. (3) define the upper and lower trailing edge thicknesses. Equation (3) also uses a general class function to define the basic profile and the shape function to create the specific shape within that geometry class. The class function is defined as:

$$
C_{N 2}^{N 1}(\psi)=\psi^{N 1} \cdot(1-\psi)^{N 2}
$$

For a general NACA type symmetric airfoil with a round nose and pointed aft end, N1 is 0.5 and N2 is 1.0 in the class function. This classifies the final shape as being within the airfoil geometry class, which forms the basis of CST airfoil representation. This means that all other airfoils represented by the CST method are derived from the class function airfoil. This is due to the fact that if the shape function equals one everywhere, the resulting airfoil is equivalent to that given by the class function. Therefore, to represent an airfoil with the CST method, N1 and N2 can be replaced with 0.5 and 1.0 respectively. To represent an airfoil, Eq. (3) then becomes:

$$
\begin{aligned}
\zeta_{U}(\psi) & =C_{1.0}^{0.5}(\psi) \cdot S_{U}(\psi)+\psi \cdot \Delta \zeta_{U} \\
\zeta_{L}(\psi) & =C_{1.0}^{0.5}(\psi) \cdot S_{L}(\psi)+\psi \cdot \Delta \zeta_{L}
\end{aligned}
$$

Many other classes exist, but the two-dimensional CST analysis will be limited to airfoils. Figure 3 displays the NACA-type airfoil as represented by the class function.

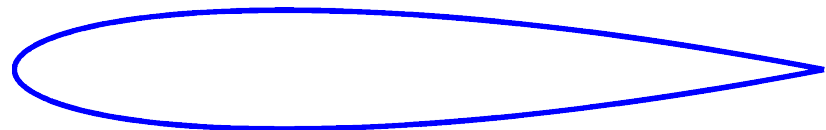

Figure 3. General airfoil defined with class function

The shape function defines the specific shape within the airfoil class. The overall shape functions for the upper and lower surfaces are as follows:

$$
\begin{aligned}
& S_{U}(\psi)=\sum_{i=0}^{N_{U}} A_{U}(i) \cdot S(\psi, i) \\
& S_{L}(\psi)=\sum_{i=0}^{N_{L}} A_{L}(i) \cdot S(\psi, i)
\end{aligned}
$$

where $\mathrm{S}$ is the component shape function and is represented by a Bernstein polynomial. A is the set of curvature coefficients to represent a given airfoil. $\mathrm{N}$ is the order of the Bernstein polynomial used for either the upper or lower surface. This is also equal to one less than the number of curvature coefficients used. The component shape function is scaled by the curvature coefficients, which determines the specific airfoil shape. The component shape function is given as the following:

$$
S(\psi, i)=K_{i}^{N} \cdot \psi^{i} \cdot(1-\psi)^{N-i}
$$


where $\mathrm{K}$ is the binomial coefficient, which is directly related to the order of the Bernstein polynomials used. The binomial coefficient is defined as:

$$
K_{i}^{n}=\frac{n !}{i !(n-i) !}
$$

Equations (5) - (9) can be combined to form the complete equations to represent the upper and lower surfaces of CST airfoils.

$$
\begin{aligned}
& \zeta_{U}(\psi)=\psi^{0.5} \cdot(1-\psi)^{1.0} \sum_{i=0}^{N_{U}}\left[A_{U}(i) \cdot \frac{N_{U} !}{i !\left(N_{U}-i\right) !} \cdot \psi^{i} \cdot(1-\psi)^{N_{U}-i}\right]+\psi \cdot \Delta \zeta_{U} \\
& \zeta_{L}(\psi)=\psi^{0.5} \cdot(1-\psi)^{1.0} \sum_{i=0}^{N_{L}}\left[A_{L}(i) \cdot \frac{N_{L} !}{i !\left(N_{L}-i\right) !} \cdot \psi^{i} \cdot(1-\psi)^{N_{L}-i}\right]+\psi \cdot \Delta \zeta_{L}
\end{aligned}
$$

Equation (10) fully describes any smooth airfoil given the correct curvature coefficients. These coefficients can be optimized to represent a known airfoil. Having an airfoil parameterized by the CST method gives an equation for the upper and lower surfaces. This allows points to be added at desired locations to refine areas such as the leading edge of an airfoil that has high curvature. Figure 4 shows some examples of parameterized airfoils to display the power of the CST parameterization method. The circles show the exact airfoil coordinates and the lines correspond to the CST airfoil surface calculated using optimized curvature coefficients.
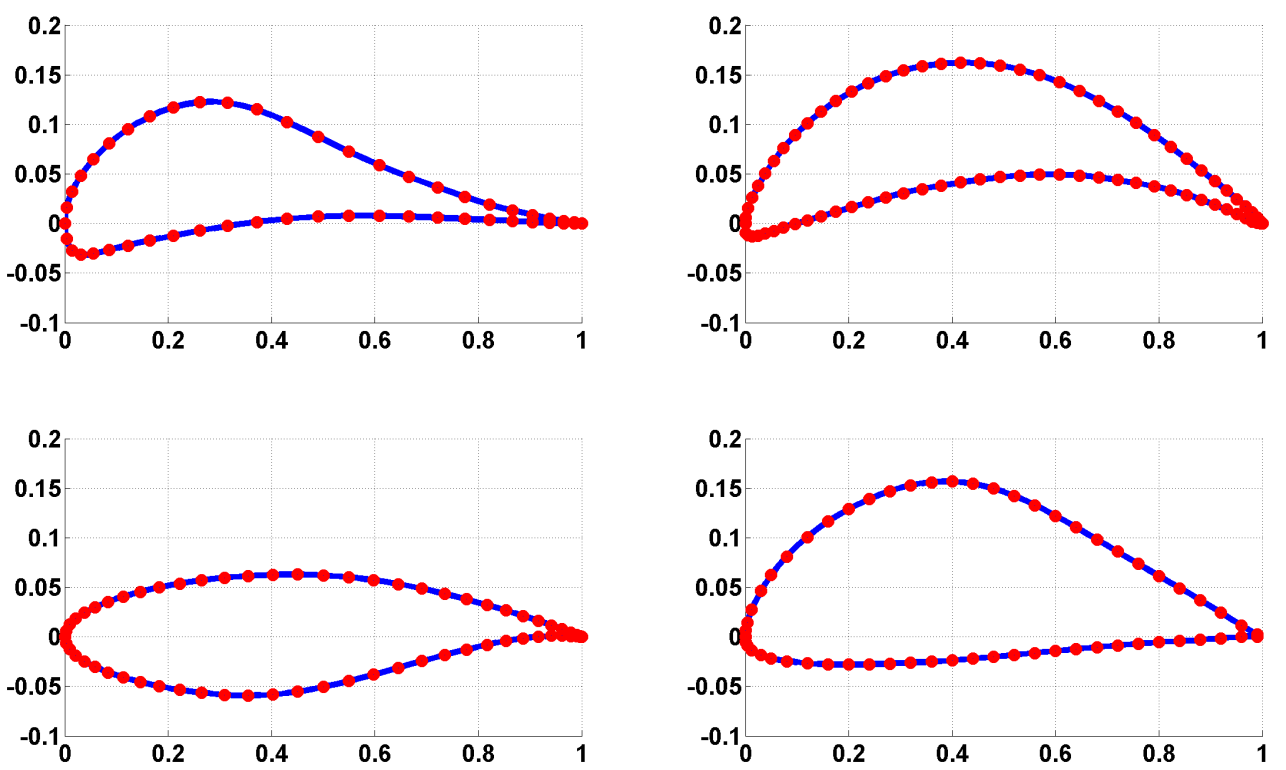

Figure 4. Airfoils parameterized using curvature coefficient optimization

In order to solve for the curvature coefficients to represent a given airfoil, Eq. (10) must be put in matrix form. This is accomplished by creating a function that isolates $\vec{A}$ since it contains the Bernstein polynomial coefficients to represent a given airfoil. Therefore, all the terms multiplied by $\vec{A}$ must be combined into a single matrix. These terms are built up as a CST geometry matrix shown below.

$$
D=\left[\begin{array}{ccc}
C_{N 2}^{N 1}[\psi(0)] \cdot K_{0}^{N} \cdot \psi(0)^{0} \cdot[1-\psi(0)]^{N-0} & C_{N 2}^{N 1}[\psi(0)] \cdot K_{1}^{N} \cdot \psi(0)^{1} \cdot[1-\psi(0)]^{N-1} & \cdots \\
C_{N 2}^{N 1}[\psi(1)] \cdot K_{0}^{N} \cdot \psi(1)^{0} \cdot[1-\psi(1)]^{N-0} & C_{N 2}^{N 1}[\psi(1)] \cdot K_{1}^{N} \cdot \psi(1)^{1} \cdot[1-\psi(1)]^{N-1} & \\
\vdots & & \ddots
\end{array}\right]
$$


Now the matrix math formulation of Eq. (10) can easily be created.

$$
\vec{\zeta}=D \cdot \vec{A}+\vec{\psi} \cdot \Delta \zeta
$$

Since $\vec{A}$ is the only unknown term for a given airfoil in Eq. (12), it can be rearranged to solve for $\vec{A}$. Since Eq. (12) is an overdetermined rectangular system unless the order of the Bernstein polynomial equals the number of points used to represent the airfoil, the pseudo inverse is used to solve for the curvature coefficients. This minimizes the least squared error between the given $\vec{\zeta}$ values and the calculated ones.

\section{III.B. Airfoil Smoothing}

The CST method was used to smooth the airfoil at each iteration in the inverse design process. The airfoil was perturbed using Eq. (2). Since these updated points do not yield a smooth airfoil, a CST curve is fit to the updated points to yield the airfoil for the next iteration of the design.

\section{Airfoil Design Results}

Three design cases are presented in the following sections to illustrate the accuracy and efficiency of the inverse design method. The cases were selected to correspond to Yu's design cases. The initial and target airfoils for each design case are a NACA 0012 and an RAE 2822, respectively. The design cases shown used CFD to calculate the airfoil pressure distributions. ICEM CFD ${ }^{8}$ was used for meshing and FLUENT $^{9}$ for solving. Similar results were obtained, but not shown here, using a panel code with integral boundary layer model for the viscous effects. Since inverse airfoil design is an iterative process, both the meshing and solution processes were automated. This was accomplished with MATLAB. ${ }^{10}$ A framework has been created to mesh a given airfoil and solve it under given flight conditions.

\section{IV.A. Subsonic Airfoil Design}

The subsonic design case is for $M_{\infty}=0.3, R e=1.0 \times 10^{7}$, and $\alpha=4.0^{\circ}$. Figure 5 shows the initial, target, and current pressure distributions, as well as pressure residuals at design iteration 5 . Figure 6 on the next page shows the initial, target, and current airfoils and the geometry residuals. Figures 7 on the following page and 8 on the next page display the same information after 20 design iterations. In Fig. 5(b) and Fig. 7(b) on the next page, $C_{p, o}$ represents the target pressure coefficient. In Fig. 6(b) on the following page and Fig. 8(b) on the next page, $y / c_{o}$ represents the target airfoil coordinate. Also, negative $x / c$ locations represent the lower surface of the airfoil and the positive $x / c$ locations represent the upper surface. Therefore, the error plots report values clockwise around the airfoil starting at the trailing edge. At iteration 5, the pressure residuals are very low everywhere except at the leading edge. The geometry residuals are also very small. They are all are below $3 \times 10^{-3}$. However, at iteration 20, the pressure distribution closely matches the target pressures. The highest error in pressure is still at the leading edge, but all the pressure residuals are below $3 \times 10^{-3}$. The geometry residuals are even better. They are all are below $1 \times 10^{-4}$.

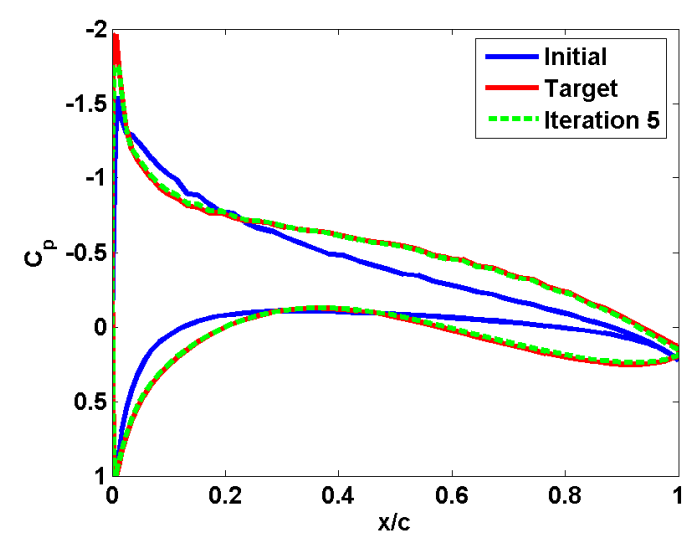

(a) Pressure Coefficient

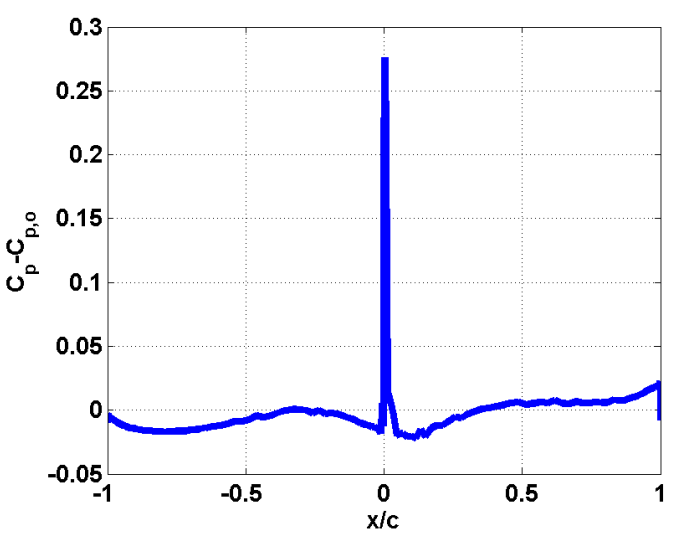

(b) Pressure Coefficient Residuals

Figure 5. Subsonic Design Pressure After 5 Iterations 


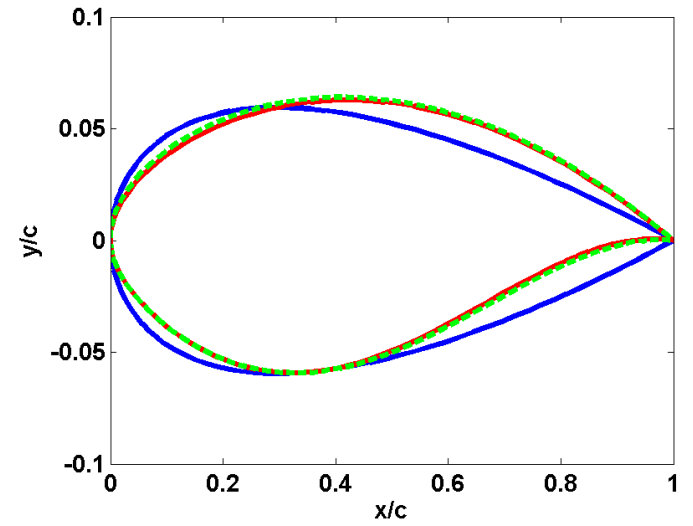

(a) Airfoil Geometry

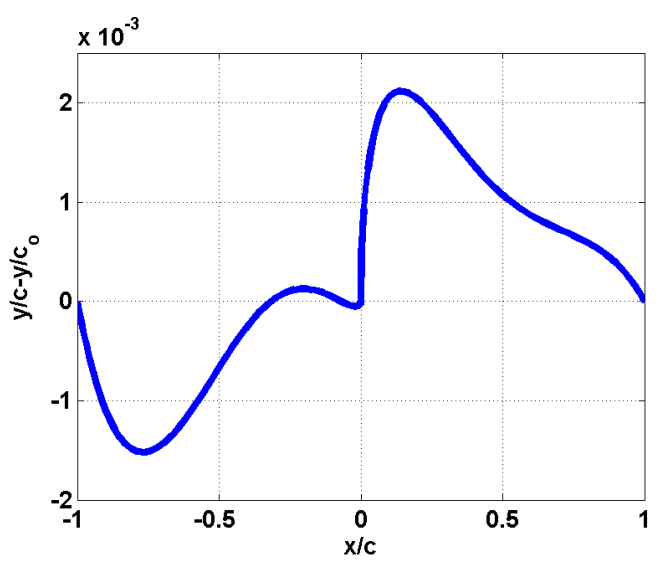

(b) Airfoil Geometry Residuals

Figure 6. Subsonic Design Geometry After 5 Iterations

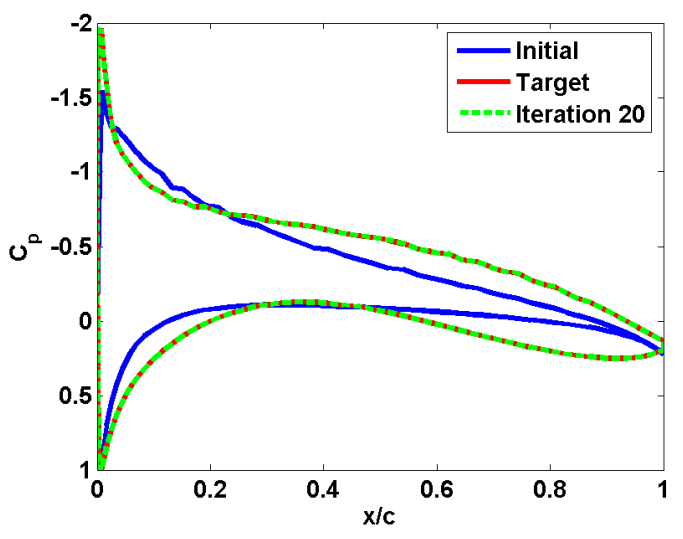

(a) Pressure Coefficient

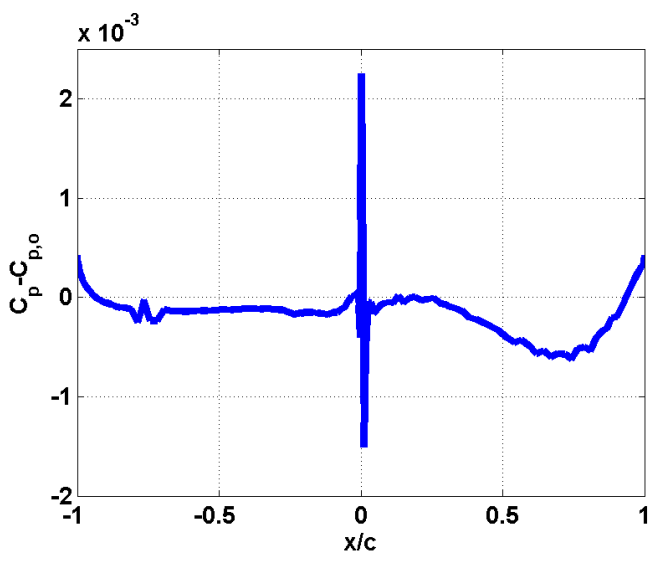

(b) Pressure Coefficient Residuals

Figure 7. Subsonic Design Pressure After 20 Iterations

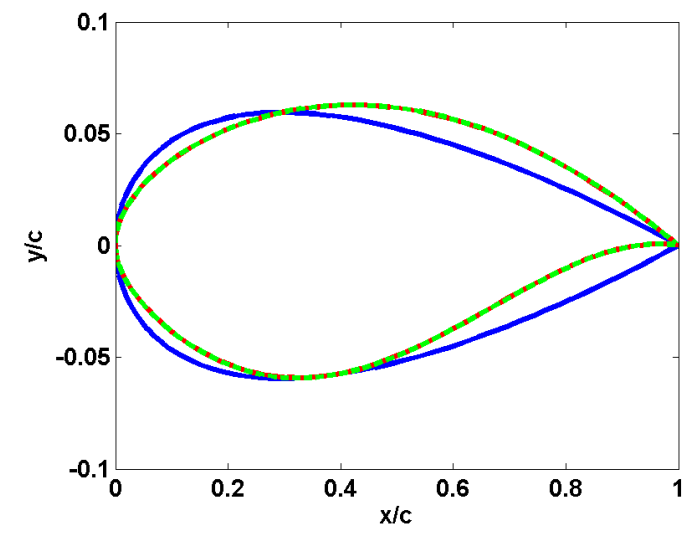

(a) Airfoil Geometry

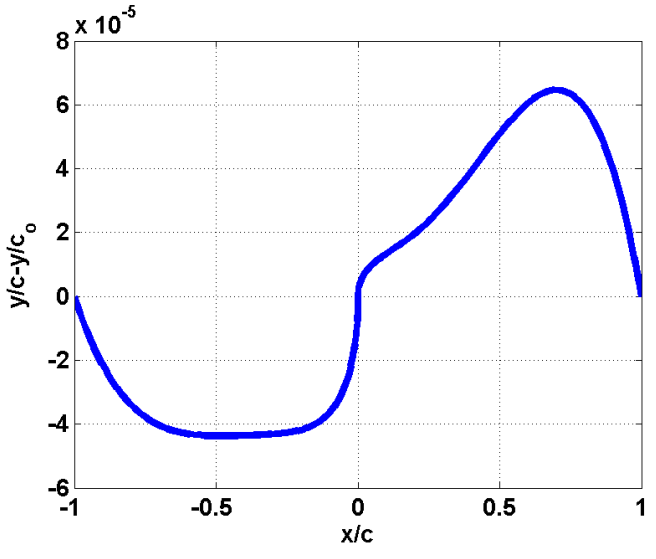

(b) Airfoil Geometry Residuals

Figure 8. Subsonic Design Geometry After 20 Iterations 
Figure 9 shows the pressure and geometry root mean square (RMS) error and maximum residual convergence. The error converges fairly steadily throughout the design process.

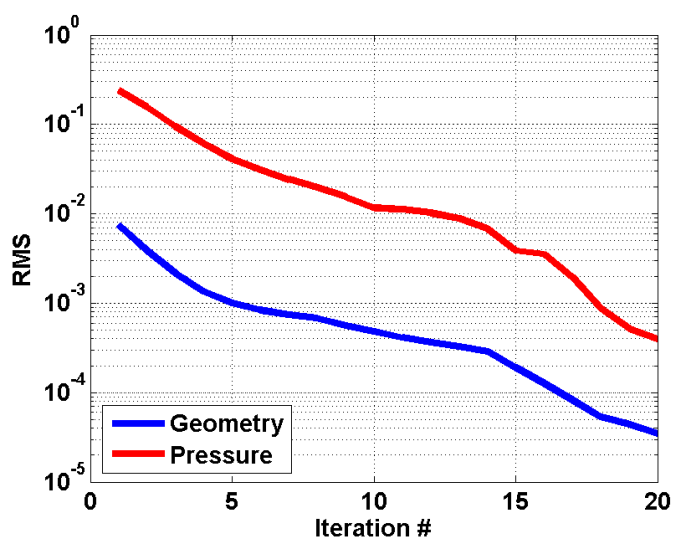

(a) RMS

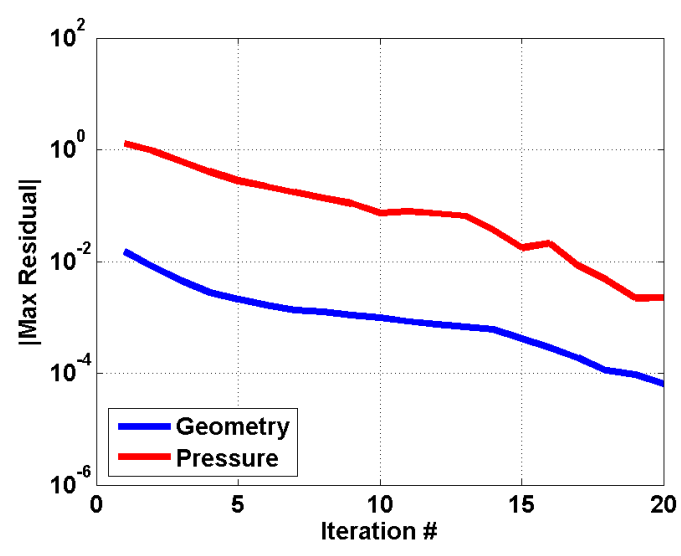

(b) Maximum Residual

Figure 9. Error Convergence for Subsonic Design

Figure 10 shows how the lift and drag coefficients converge to the target values. The lift coefficient converges very quickly. It matches to within $5 \%$ of the target value in 5 design iterations and is nearly exact by 8 design iterations. However, the drag coefficient takes longer to converge and varies much more throughout the design than the lift coefficient. It does not display the smooth convergence of the lift coefficient. It matches to within $2 \%$ by the second design iteration, but varies within that region for the remainder of the design. The drag coefficient nearly matches the target value at the end of the design.

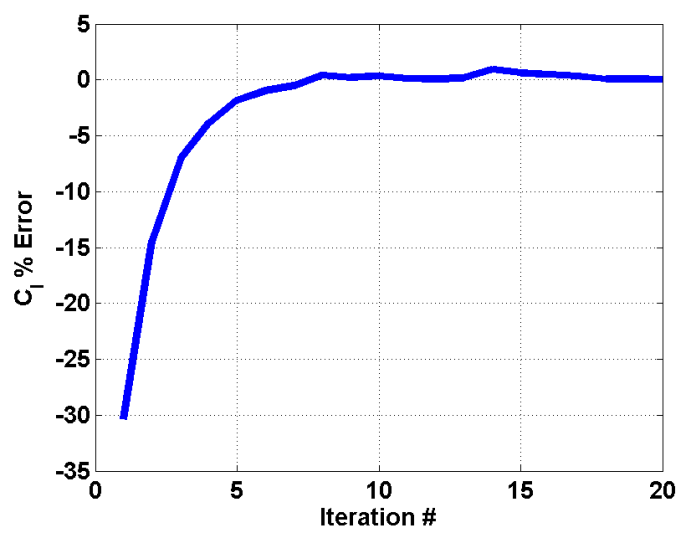

(a) Lift Coefficient

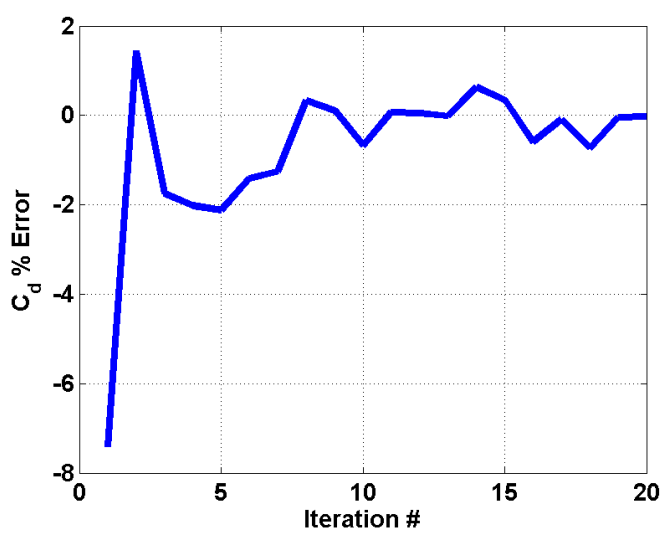

(b) Drag Coefficient

Figure 10. Lift and Drag Coefficient Convergence for Subsonic Design

\section{IV.B. High Subsonic Airfoil Design}

The high subsonic design case is for $M_{\infty}=0.6, R e=1.0 \times 10^{7}$, and $\alpha=1.5^{\circ}$. Figure 11 on the following page through Fig. 14 on page 10 show the high subsonic inverse design results at iterations 5 and 20. The slight bumps in the pressure distributions are believed to be a numerical artifact and is under investigation. Again, by iteration 5, the pressure residuals are all very low except for at the leading edge. After 20 design iterations, all pressure residuals are within $2 \times 10^{-3}$ and all geometry residuals are within $8 \times 10^{-5}$. 


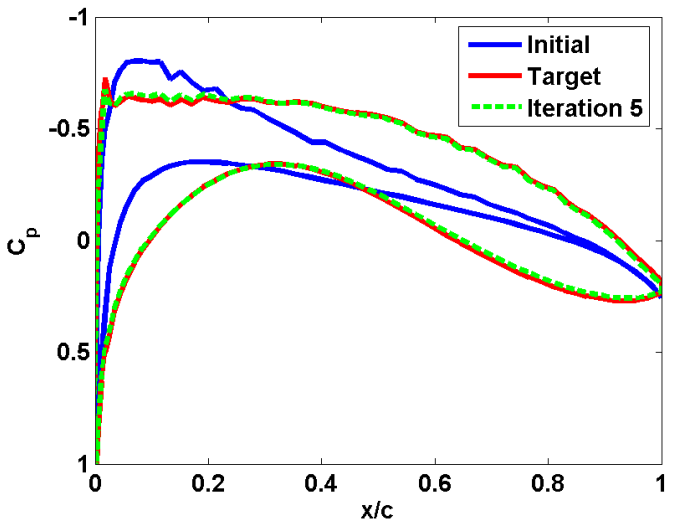

(a) Pressure Coefficient

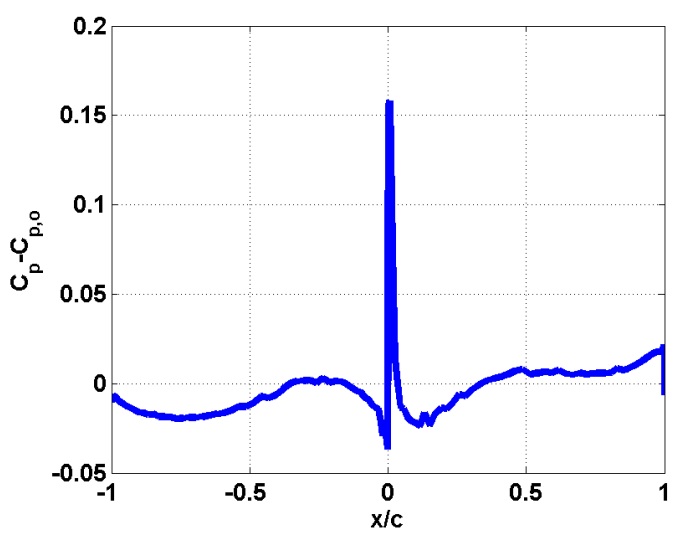

(b) Pressure Coefficient Residuals

Figure 11. High Subsonic Design Pressure After 5 Iterations

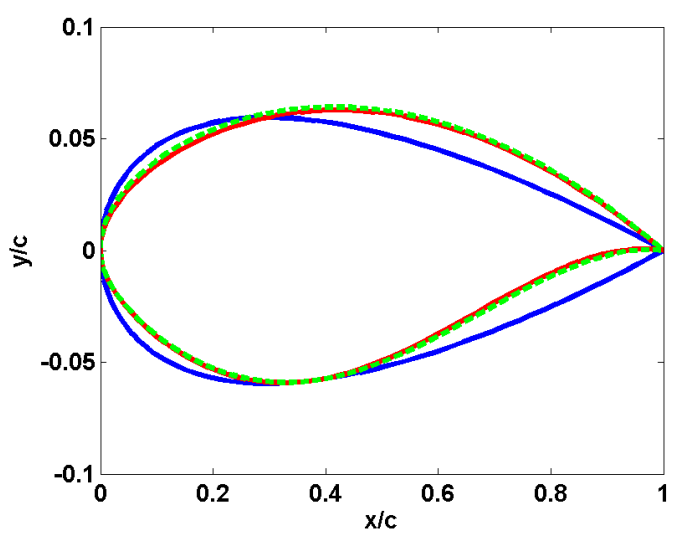

(a) Airfoil Geometry

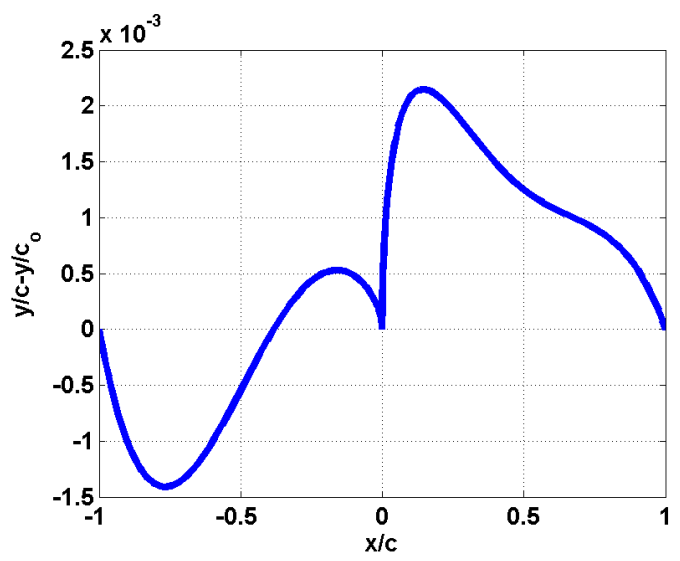

(b) Airfoil Geometry Residuals

Figure 12. High Subsonic Design Geometry After 5 Iterations

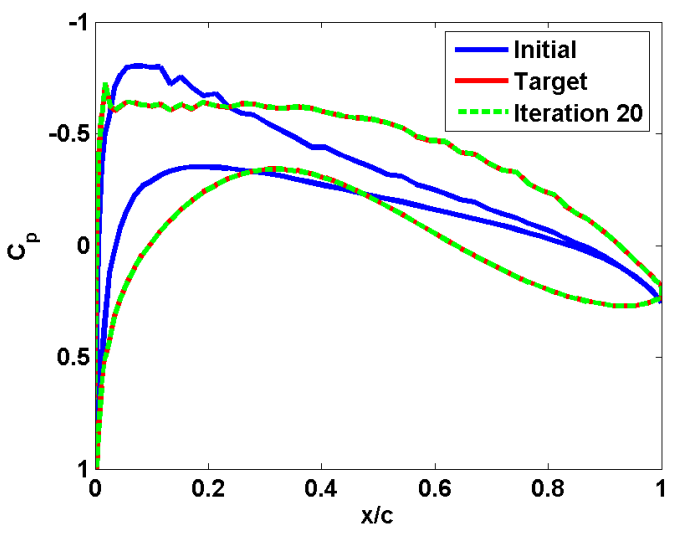

(a) Pressure Coefficient

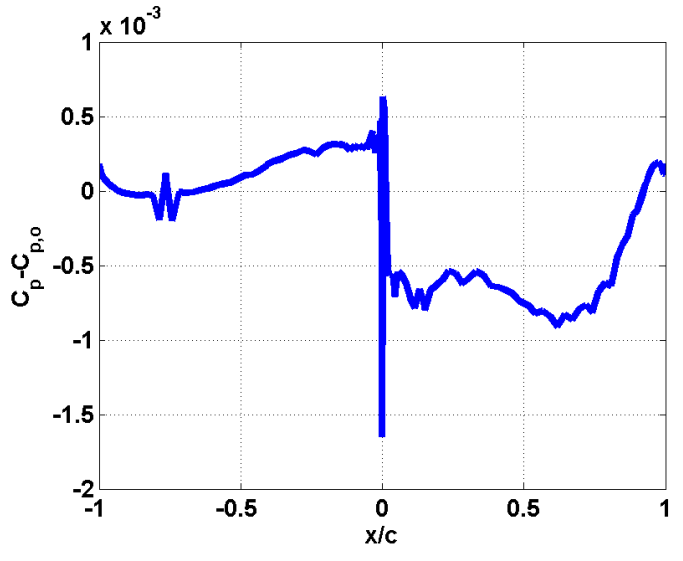

(b) Pressure Coefficient Residuals

Figure 13. High Subsonic Design Pressure After 20 Iterations 


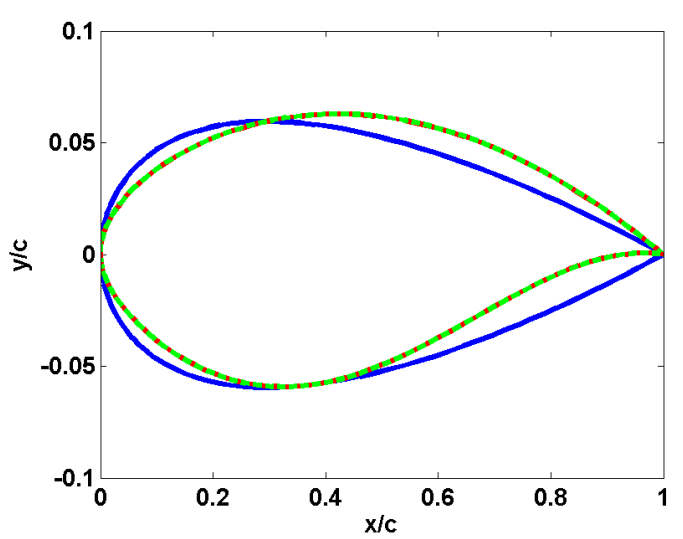

(a) Airfoil Geometry

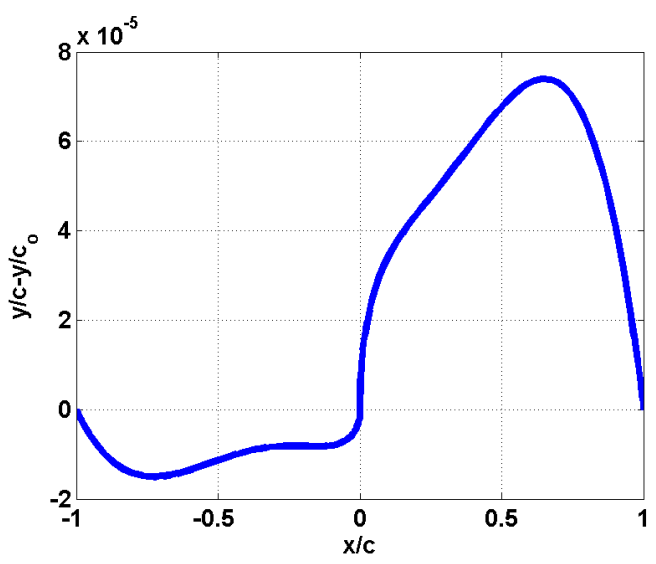

(b) Airfoil Geometry Residuals

Figure 14. High Subsonic Design Geometry After 20 Iterations

Figure 15 shows the error convergence for the high subsonic design case. Again, the error decreases rather steadily throughout the design.

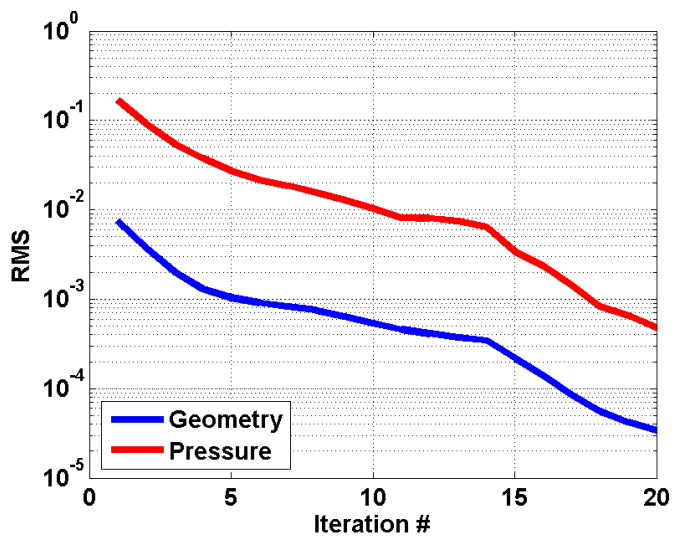

(a) RMS

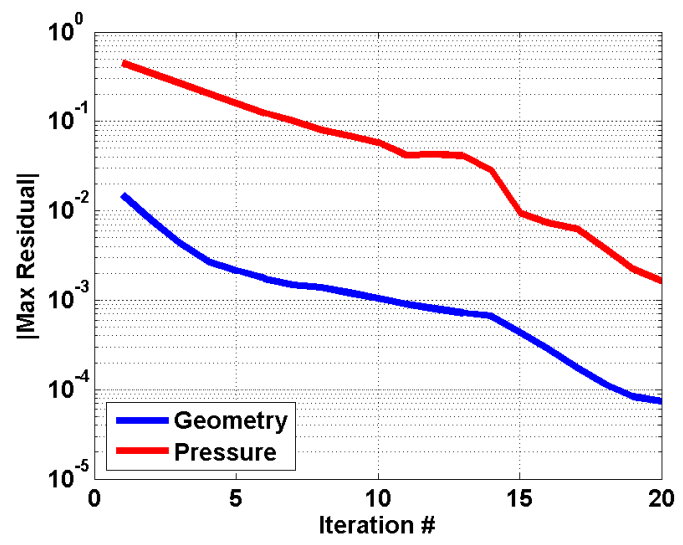

(b) Maximum Residual

Figure 15. Error Convergence for High Subsonic Design 
Figure 16 shows the lift and drag coefficient convergence for the high subsonic design case. It converges fairly smoothly to the target value. The lift coefficient is within $5 \%$ of the target value after 5 design iterations and nearly matches by iteration 8 . Again, the drag coefficient takes much longer to converge than the lift coefficient. It overshoots and gradually decreases to the target value. It gets to within $2 \%$ of the target value by iteration 9 , but does not match until iteration 20 .

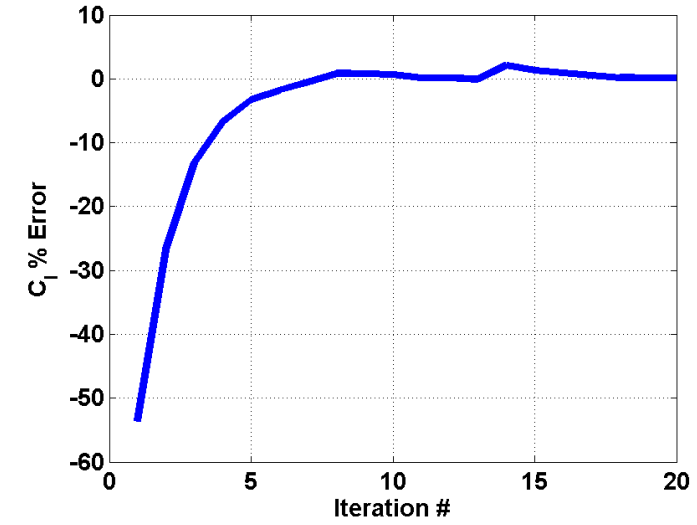

(a) Lift Coefficient

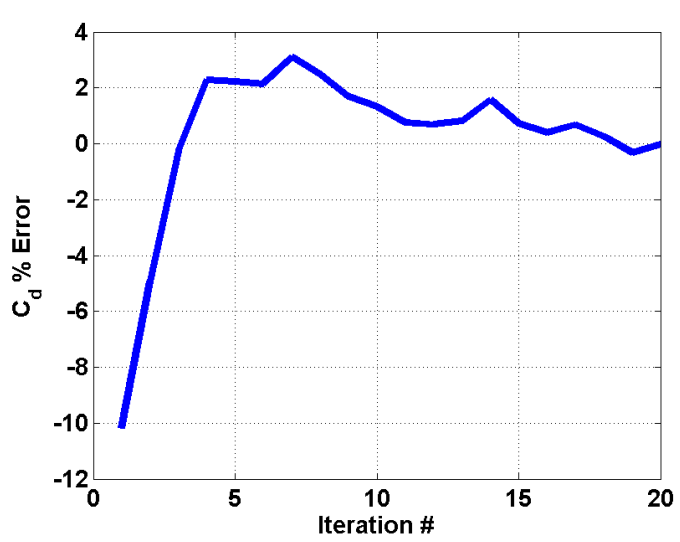

(b) Drag Coefficient

Figure 16. Lift and Drag Coefficient Convergence for High Subsonic Design

\section{IV.C. Transonic Airfoil Design}

The transonic design case is for $M_{\infty}=0.715, R e=1.0 \times 10^{7}$, and $\alpha=2.3^{\circ}$. Figure 17 through Fig. 20 on the next page show the inverse design results at iterations 5 and 16 . The convergence is not as good due to the presence of a shock on the upper surface. By iteration 5, the lower surface is modeled well. However, there is much more error in the pressure and geometry on the upper surface because of the shock. Iteration 16 had the lowest error of the design process. The pressure jump across the shock is captured well. The maximum pressure residual is still high compared to the previous two design cases, but the geometry residuals are all within $2 \times 10^{-4}$. The highest pressure residuals do not occur at the leading edge as in the previous two cases. The highest error in pressure occurs aft of the leading edge where the bumps in the pressure distribution occur. The error may greatly reduce if these bumps are eliminated.

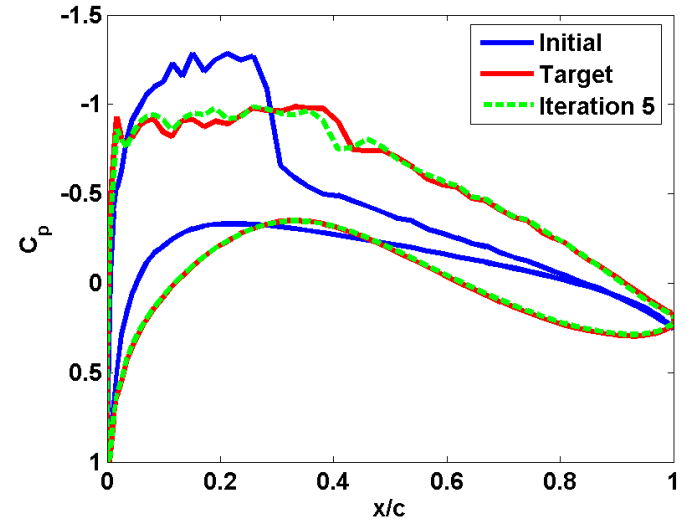

(a) Pressure Coefficient

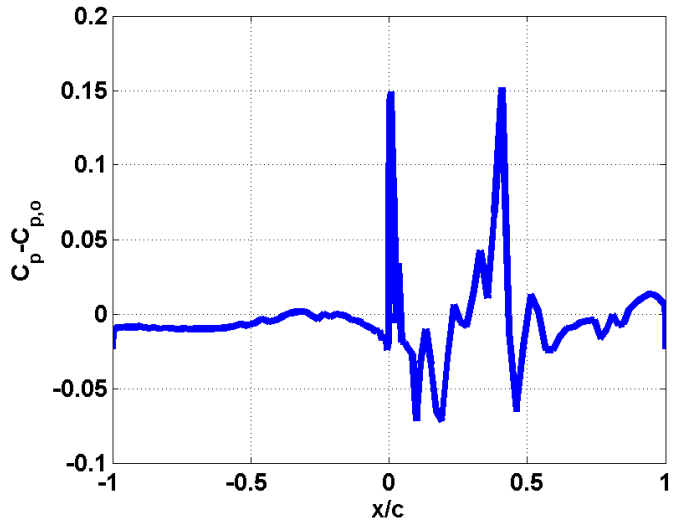

(b) Pressure Coefficient Residuals

Figure 17. Transonic Design Pressure After 5 Iterations 


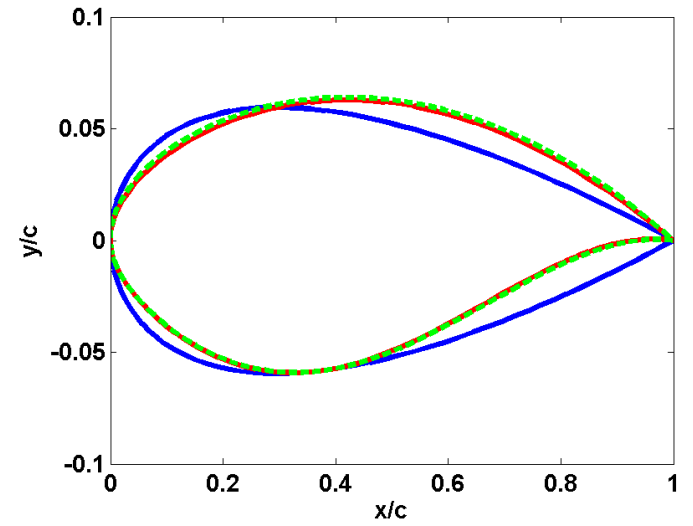

(a) Airfoil Geometry

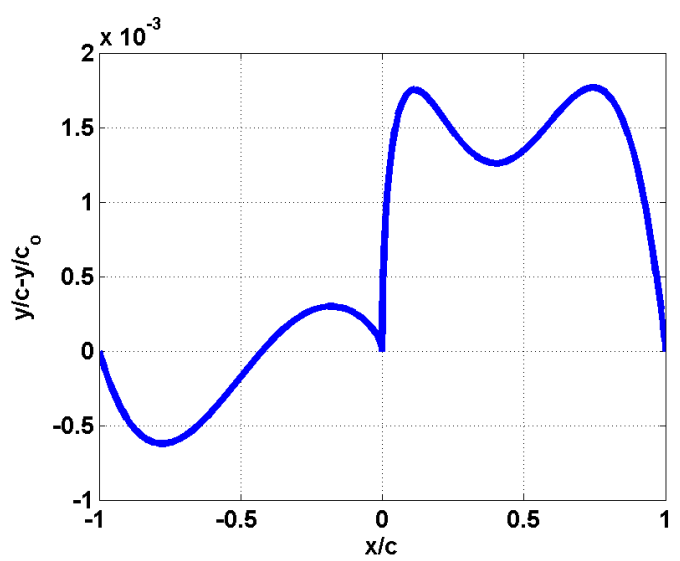

(b) Airfoil Geometry Residuals

Figure 18. Transonic Design Geometry After 5 Iterations

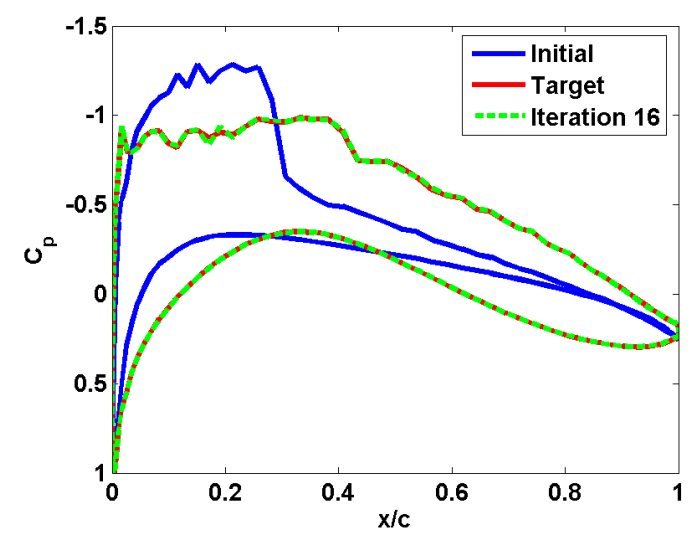

(a) Pressure Coefficient

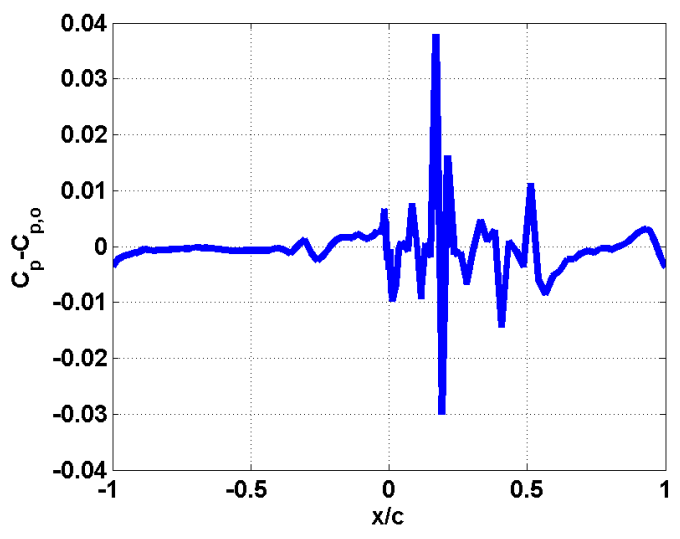

(b) Pressure Coefficient Residuals

Figure 19. Transonic Design Pressure After 16 Iterations

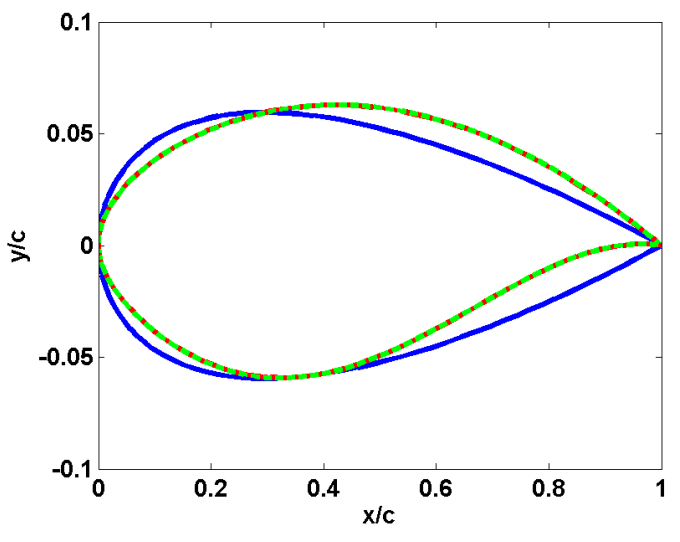

(a) Airfoil Geometry

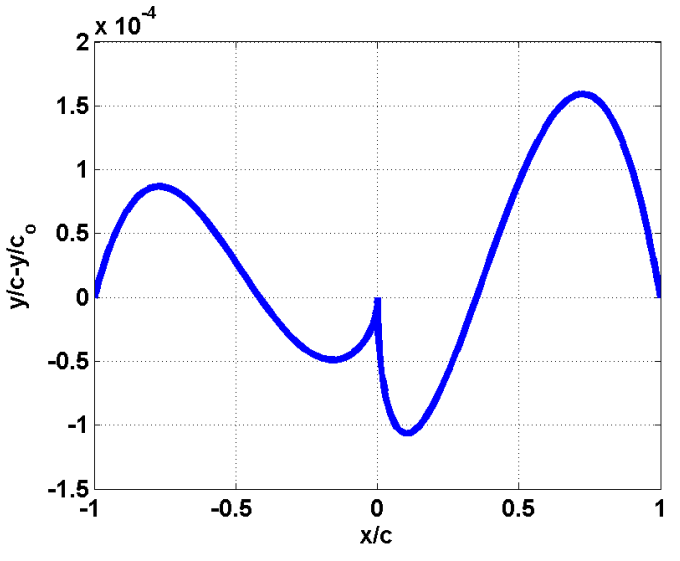

(b) Airfoil Geometry Residuals

Figure 20. Transonic Design Geometry After 16 Iterations 
Figure 21 shows the error convergence for the transonic design case. The error decreases much slower than the previous two design cases. There are also several iterations that increase the error. This is most likely due to the airfoil oscillating around the target airfoil. Careful selection of the relaxation factor may decrease these oscillations and therefore improve the error of the design.

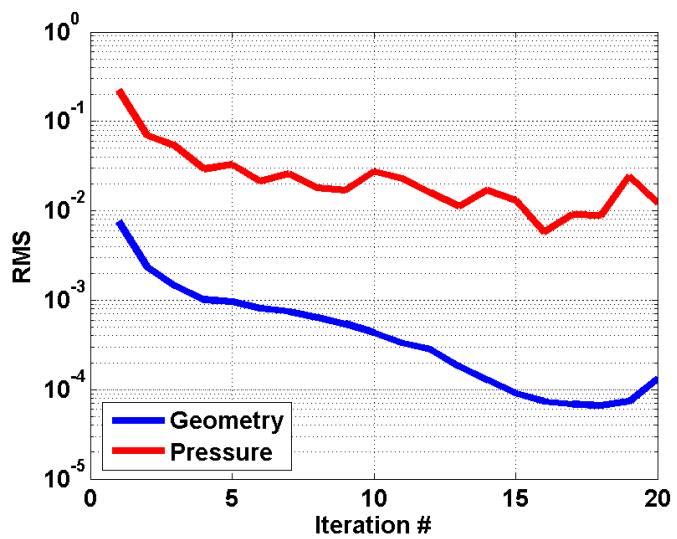

(a) RMS

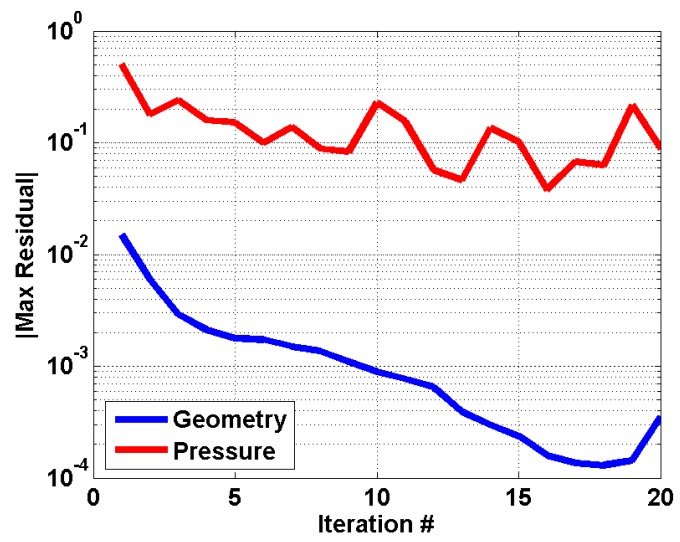

(b) Maximum Residual

Figure 21. Error Convergence for Transonic Design

Figure 22 shows the lift and drag coefficient convergence for the transonic design case. The lift coefficient still converges quickly despite the additional error in the geometry due to the shock. It reaches $1 \%$ of the target lift coefficient by iteration 5 and maintains that accuracy for the remainder of the design with the exception of two iterations where the lift coefficient increases slightly. Again, the drag coefficient takes longer to converge than the lift coefficient. It overshoots and slowly decreases. It stays within $2 \%$ of the target value after 10 design iterations and is well within $1 \%$ by the end of the design.

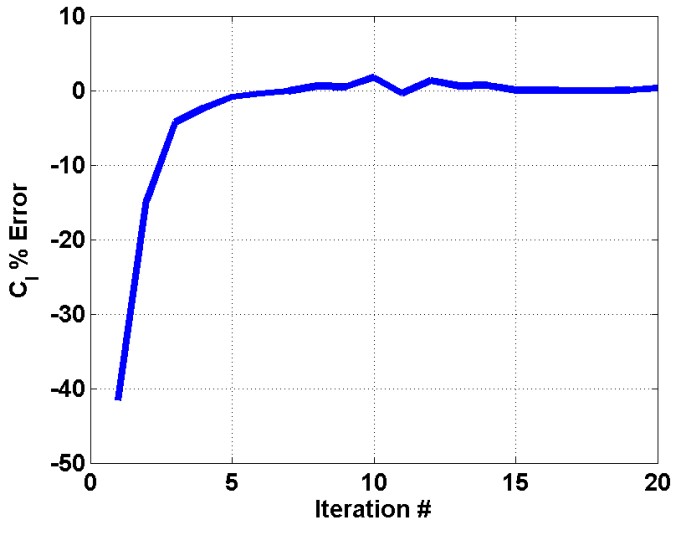

(a) Lift Coefficient

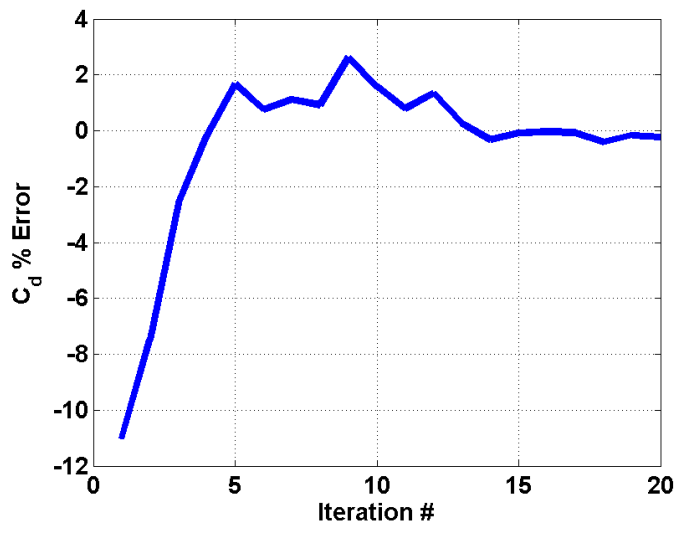

(b) Drag Coefficient

Figure 22. Lift and Drag Coefficient Convergence for Transonic Design

\section{Conclusions}

The inverse airfoil design method presented is simple, efficient, and accurate. It can be used with any fidelity model since it only requires a current and target pressure distribution. It has been shown to be accurate for both subsonic and transonic flows. The method is based on how the desired change in pressure relates to the required change in the airfoil shape. Increasing pressure requires the airfoil to be perturbed with a negative outward facing normal and decreasing pressure requires the airfoil to be perturbed with a positive outward facing normal. Therefore, the pressure residuals have the same sign as the required normal vector and this vector can simply be scaled to modify the current airfoil in the design process. Each coordinate of the airfoil was perturbed along its normal vector with the sign of the vector equal to the pressure residual at 
that point. The updated airfoil was then smoothed using the CST parameterization method. The highest pressure residuals occurred at the leading edge. However, with the exception of the transonic case, these residuals became very small. The pressure residuals were within $3 \times 10^{-3}$ for the subsonic case and within $2 \times 10^{-3}$ for the high subsonic case at the end of the design. The highest pressure residuals for the transonic case occurred where the bumps in the pressure distribution were located, not the leading edge. Eliminating these bumps may greatly reduce the error for the transonic case.

\section{Future Work}

A study will be conducted on the effects of the order of the CST curve on the accuracy and efficiency of the inverse design process. The process of calculating perturbations using the pressure residuals need to be studied further to see if they hold for a supersonic case or for a transonic case with a stronger shock than the transonic case investigated in this paper. The relaxation factors used also need to be studied to maximize the efficiency of the method. Ways to reduce the pressure residuals at the leading edge will also be investigated. This will keep the error over the entire airfoil within a much narrower band.

\section{Acknowledgements}

This work was funded as part of a NASA Research Announcement award under Contract \#NNL07AA55C with Craig Hange and Clif Horne as the technical monitors. The authors wish to thank Brenda Kulfan for her fruitful discussions about the CST method.

\section{References}

${ }^{1}$ Obayashi, S. and Takanashi, S., "Genetic Optimization of Target Pressure Distributions for Inverse Design Methods," AIAA Journal, Vol. 34, No. 5, 1996, pp. 881-886.

2 vanEgmond, J. A., "Numerical Optimization of Target Pressure Distributions for Subsonic and Transonic Airfoil Design," Computational Methods for Aerodynamic Design (Inverse) and Optimization, AGARD CP 463, March 1990.

${ }^{3}$ Takanashi, S., "Iterative Three-Dimensional Transonic Wing Design Using Integral Equations," Journal of Aircraft, Vol. 22, No. 8, 1985, pp. 823-829.

${ }^{4}$ Barger, R. L. and Brooks, C. W. J., "A Streamline Curvature Method for Design of Supercritical and Subcritical Airfoils," Tech. rep., NASA TN D-7770, September 1974.

${ }^{5} \mathrm{Yu}$, J., Paraschivoiu, I., and Saeed, F., "Iterative Inverse Design Method Based on Streamline Equations," Journal of Aircraft, Vol. 41, No. 4, 2004, pp. 821-828.

${ }^{6}$ Kulfan, B. M., "A Universal Parametric Geometry Representation Method - 'CST'," $45^{\text {th }}$ AIAA Aerospace Sciences Meeting and Exhibit, AIAA, 8-11 January 2007.

${ }^{7}$ Lane, K. and Marshall, D., "A Surface Parameterization Method for Airfoil Optimization and High Lift 2D Geometries Utilizing the CST Methodology," 4 $7^{\text {th }}$ AIAA Aerospace Sciences Meeting and Exhibit, AIAA, 5-8 January 2009.

${ }^{8}$ Ansys Inc., Canonsburg, PA, ICEM CFD, 11th ed., 2008.

${ }^{9}$ Ansys Inc., Lebanon, NH, FLUENT, 6th ed., 2008.

${ }^{10}$ The Mathworks Inc., Natick, MA, MATLAB, 7th ed., 2008. 